\title{
Evaluation of Bovine Serum Albumin as a Fluorescence Enhancement Reagent in Liquid Chromatography
}

\author{
Junichi Ishida, Kazutomo Abe, Masaru NaKamura and Masatoshi Yamaguchi ${ }^{\dagger}$ \\ Faculty of Pharmaceutical Sciences, Fukuoka University, \\ Nanakuma, Johna, Fukuoka 814-80, Japan
}

\begin{abstract}
Bovine serum albumin (BSA) was evaluated as a fluorescence enhancement reagent using 41 fluorescent compounds. The degree of the enhancement was affected by basic fluorescent skeletons and by side-chains of the compounds. BSA was found to be a fairly selective fluorescence enhancement reagent for some compounds such as dansyl amino acids. BSA was applied for the determination of dansyl amino acids by postcolumn high-performance liquid chromatography with fluorescence enhancement detection. Detection limits for the amino acids were $34-169 \mathrm{fmol}$ on column at a signal-to-noise ratio of three. Dansyl amino acids could be measured $8-109$ times more sensitively with the enhancement detection method than with the conventional fluorescence detection.
\end{abstract}

Keywords Bovine serum albumin, fluorescence enhancement, postcolumn high-performance liquid chromatography, dansyl amino acid

Various methods such as spectrophotometric, fluorometric, laser-induced fluorimetric and chemiluminometric detection, have been used for high-performance liquid chromatographic (HPLC) analysis. In fluorometric methods, many derivatization reagents have been developed for the effective use in its high sensitivity. ${ }^{1,2}$ On the other hand, fluorescence properties (intensity, wavelength and polarization) are known to vary with the chemical environment around fluorescent compounds and such changes are used for selective analysis. For example, the enhancement effect of cyclodextrins for some fluorescent compounds has been used successfully for HPLC detection (fluorescence enhancement detection). ${ }^{3,4}$

Recently, we found that dansyl undecanoic acid, which has a fluorescent probe and a fatty acid residue, binds selectively to bovine serum albumin (BSA) and fatty acid binding proteins (FABP) to increase dramatically the fluorescence with a blue shift of the emission wavelength. ${ }^{5}$ The fluorescence enhancement was caused by the combining of dansyl undecanoic acid to the hydrophobic binding site(s) on the proteins.

In this study, we have evaluated BSA as a selective fluorescence enhancement reagent for various fluorescent compounds. The enhancement effect of BSA was dependent on the structure of the basic probe and on the side-chain of the fluorescent compounds. Consequently, BSA was found to be an excellent reagent as a fluorescence enhancer for some compounds, such as dansyl (DNS) amino acids. Based on this finding, we have developed an HPLC method, with fluorescence enhancement detection, using BSA as a postcolumn modification reagent. DNS amino acids were used as model compounds for the HPLC studies.

\section{Experimental}

\section{Reagents and solutions}

Unless otherwise stated, all chemicals and solvents were of analytical-reagent grade and were used without further purification. Water was deionized, distilled and further purified using a Milli-Q II system (Japan Millipore, Tokyo, Japan). BSA (RIA Grade, Fraction V) and DNS amino acids were purchased from Sigma (St. Louis, MO, USA). 11-[(5-Dimethylaminonaphthalene-1-sulfonyl)amino]undecanoic acid (DNS-undecanoic acid), 12-[ $N$-methyl- $N$-(7-nitrobenz-2-oxa-1,3diazol-4-yl)amino] octadecanoic acid (NBD-stearic acid), 1-pyreneundecanoic acid (pyrene-undecanoic acid), 11[ $N$-(7-diethylamino-3-coumarinoyl)amino]undecanoic acid (coumarin-undecanoic acid) and 11-(9-anthroyloxy)undecanoic acid (anthracene-undecanoic acid) were obtained from Molecular Probes (Junction City, OR, USA). Other fluorescent compounds were purchased from Wako (Tokyo, Japan). BSA solutions ( $1 \mathrm{mM}$ and $60 \mu \mathrm{M}$ ) were prepared in water and $100 \mathrm{mM}$ phosphate buffer ( $\mathrm{pH} 9.0$ ), respectively, and were used within a week. Stock solutions of the fluorescent compounds $(10 \mathrm{mM})$ were prepared in methanol and diluted further with $10 \mathrm{mM}$ phosphate buffer ( $\mathrm{pH} 7.0$ ) before use to give the required concentrations.

\footnotetext{
$\dagger$ To whom correspondence should be addressed.
} 
Manual procedure for evaluation of BSA as a fluorescence enhancer

For the preparation of a test solution, $40 \mu \mathrm{l}$ each of the fluorescent compound solution $(0.1 \mathrm{mM})$ and the BSA solution ( $1 \mathrm{mM}$ ) were added to $4.0 \mathrm{ml}$ of $10 \mathrm{mM}$ phosphate buffer ( $\mathrm{pH} 7.0)$. The reagent blank solution was prepared in the same way except that the BSA solution was replaced with water. The uncorrected fluorescence spectra and intensities of the test and blank solutions (BSA-bound and unbound probes, respectively) were measured by means of a Hitachi $650-60$ spectrofluorometer with silica cells $(10 \times 10 \mathrm{~mm})$; spectral bandwidths of $5 \mathrm{~nm}$ were used for both the excitation and the emission monochromators. The fluorescence intensities of the test and blank solutions ( $I_{\text {test }}$ and $I_{\text {blank, }}$, respectively) were measured at the wavelengths shown in Tables 1 and 2. The fluorescence enhancement factor (FEF) was defined by the equation below:

$$
\mathrm{FEF}=I_{\text {test }} / I_{\text {blank }}
$$

\section{Postcolumn HPLC system with BSA}

Sample solutions of DNS amino acids were applied onto the following HPLC system. Figure 1 shows a schematic flow diagram of the HPLC-fluorescence enhancement detection system. Chromatography was performed with a Tosoh (Tokyo, Japan) CCPM highperformance liquid chromatograph $\left(\mathbf{P}_{1}\right)$ equipped with a Rheodyne 7125 syringe-loading sample injector valve (I) $(20 \mu$ loop). Chromatograms were recorded with UNICORDER U-228 (REC). DNS-amino acids were separated on a TSKgel QAE 2SW strong anion exchange column $(5 \mu \mathrm{m}, 250 \times 4.6 \mathrm{~mm}$ i.d.; Tosoh) by isocratic elution with $50 \mathrm{mM}$ phosphate buffer $(\mathrm{pH} 2.3)$ as eluent (E). The flow rate of the eluent was $0.8 \mathrm{ml} \mathrm{min}^{-1}$. The column temperature was ambient $\left(18-25^{\circ} \mathrm{C}\right)$.

The eluate from the HPLC column was mixed with the $60 \mu \mathrm{M}$ BSA solution prepared in $100 \mathrm{mM}$ phosphate buffer ( $\mathrm{pH}$ 9.0) by the T-type mixing device (M) delivered by a Tosoh CCPE-II pump $\left(\mathrm{P}_{2}\right)$. The flow rate of the BSA solution was $0.5 \mathrm{ml} \mathrm{min}^{-1}$. The enhanced fluorescence was monitored by a Hitachi F-1000 fluorescence detector (D) equipped with a $12 \mu \mathrm{l}$ flow cell, operated at an excitation wavelength of $345 \mathrm{~nm}$ and an emission

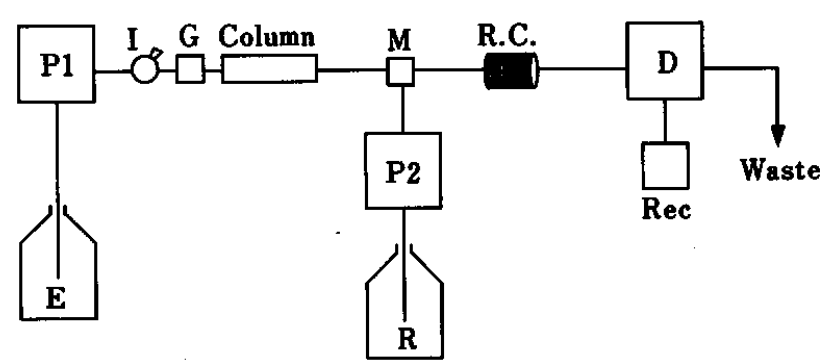

Fig. 1 Schematic flow diagram of postcolumn HPLC system with fluorescence enhancement detection. P1 \& P2, HPLC pumps; I, injection valve $(20 \mu \mathrm{l}) ; \mathrm{D}$, fluorescence detector; Column, TSKgel QAE-2SW $(250 \times 4.6 \mathrm{~mm}$ i.d. $)$; R.C., reaction coil $(5 \mathrm{~m} \times 0.5 \mathrm{~mm}$ i.d.); $\mathrm{M}$, mixing tee; Rec, recorder; E, mobile phase; $R$, postcolumn reagent solution

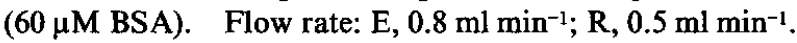

wavelength of $497 \mathrm{~nm}$. Stainless-steel tubing $(0.5 \mathrm{~mm}$ i.d.) was used for the HPLC system.

Calibration graphs for DNS amino acids were established by using the sample solutions containing 1.5 pmol $-2.5 \mathrm{nmol}$ each $\mathrm{ml}^{-1}$ of DNS amino acids.

\section{Results and Discussion}

\section{Fluorescence enhancement by BSA}

In the previous work, we reported that DNS undecanoic acid exhibited marked enhancement of fluorescence upon combining with BSA. ${ }^{5}$ The enhancement might be due to the hydrophobic interaction between the fluorescence probe and the binding site(s) on BSA; the probe was confirmed to increase remarkably the fluorescence in non-polar organic solvents. In the present work, various fluorescent compounds were examined for the enhancement study of BSA.

Twenty compounds containing various fluorescent probes were selected for the evaluation of BSA as a fluorescence enhancer. Of these, six compounds described in Table 1 showed enhanced fluorescence. Table 1 shows the fluorescence excitation and emission maxima of the BSA-bound and unbound compounds

Table 1 Fluorescence excitation and emission maxima of BSA-bound and unbound fluorescent compounds, and the fluorescence enhancement factors

\begin{tabular}{llllllr}
\hline \multirow{2}{*}{ Compound } & \multicolumn{2}{c}{ BSA-bound } & & \multicolumn{2}{c}{ Unbound } & \multirow{2}{*}{ FEF $^{\mathrm{a}}$} \\
\cline { 2 - 3 } & $\lambda_{\mathrm{ex}}$ & $\lambda_{\mathrm{em}}$ & & $\lambda_{\mathrm{ex}}$ & $\lambda_{\mathrm{em}}$ & \\
\hline 1,8-ANS & 378 & 472 & & 370 & 515 & 1286 \\
Anthracene-undecanoic acid & 365 & 449 & & 363 & 456 & 18 \\
Coumarin-undecanoic acid & 432 & 466 & & 427 & 477 & 15 \\
DNS-undecanoic acid & 345 & 497 & & 335 & 557 & 34 \\
NBD-stearic acid & 469 & 529 & & 469 & 553 & 109 \\
Pyrene-decanoic acid & 345 & 379 & & 334 & 380 & 4 \\
\hline
\end{tabular}

a. Fluorescence enhancement factors $(\mathrm{FEF})=I_{\text {test }} / I_{\text {blank. }}$. 
Table 2 Fluorescence excitation and emission maxima of the DNS fluorescent probes combined with BSA, and the fluorescence enhancement factors

\begin{tabular}{|c|c|c|c|}
\hline DNS compound & $\lambda_{\mathrm{ex}}$ & $\lambda_{\mathrm{em}}$ & FEF $^{\mathbf{a}}$ \\
\hline DNS-undecanoic acid & 345 & 497 & 34 \\
\hline DNS-Ala & 352 & 488 & 250 \\
\hline DNS-Arg & 340 & 525 & 3 \\
\hline DNS-Asn & 354 & 489 & 189 \\
\hline DNS-Asp & 348 & 488 & 206 \\
\hline DNS-Cys & 352 & 490 & 235 \\
\hline DNS-GIn & 354 & 489 & 51 \\
\hline DNS-Glu & 346 & 502 & 88 \\
\hline DNS-Gly & 351 & 490 & 229 \\
\hline DNS-Ile & 355 & 501 & 94 \\
\hline DNS-Leu & 354 & 500 & 86 \\
\hline DNS-Lys (pH 3.0) & 295 & 593 & 5 \\
\hline$(\mathrm{pH} \quad 7.0)$ & 335 & 537 & 3 \\
\hline$(\mathrm{pH} 10.0)$ & 340 & 503 & 8 \\
\hline DNS-Met & 350 & 488 & 149 \\
\hline DNS-Phe & 350 & 488 & 219 \\
\hline DNS-Pro & 355 & 489 & 363 \\
\hline DNS-Ser & 354 & 489 & 270 \\
\hline DNS-Thr & 355 & 490 & 179 \\
\hline DNS-Trp & 355 & 484 & 266 \\
\hline DNS-Tyr & 364 & 518 & 34 \\
\hline DNS-Val & 350 & 490 & 141 \\
\hline DNS-nolvaline & 356 & 490 & 210 \\
\hline DNS-L- $\alpha$-amino-butyric acid & 355 & 487 & 264 \\
\hline DNS-citrulline & 352 & 491 & 42 \\
\hline
\end{tabular}

a. Fluorescence enhancement factors $(\mathrm{FEF})=I_{\text {test }} / I_{\text {blank }}$.

and the FEF values. Five fluorescent probes containing a fatty acid residue and 8-anilino-1-naphthalenesulfonic acid sodium salt (1,8-ANS) have a hydrophobic structure and an anion charge in their molecules. 1,8-ANS enhanced the fluorescence about 1286 times by binding with BSA. 1,8-ANS is well-known to increase the fluorescence in a hydrophobic environment. ${ }^{6}$ On the other hand, the other compounds (acridine, acridine orange, azure $B$, safranin $O$, naphthalene, methylene violet, fluorescein, fluoresceinamine, maprotiline, triamterene, hydrochlorothiazide, acetazolamide and caffeine) did not increase their fluorescence when treated according to the manual procedure described in the Experimental section.

The effect of the side-chain of the fluorescent probes on the FEF was examined using various DNS amino acids (Table 2). The fluorescence intensities of all the DNS derivatives tested, except for DNS-L-arginine (DNS-Arg) and DNS-L-lysine (DNS-Lys), were increased 34-363 times by the effect of BSA. DNS-Arg and DNS-Lys showed only three times enhanced fluorescence by interaction with BSA. These derivatives have both (anionic and cationic) charges in their molecules. To examine the effect of the charges, FEF of DNS-Lys was determined at various $\mathrm{pHs}$. FEF at alkaline $\mathrm{pH}$ $(\mathrm{pH}=10)$ was 2.5 times greater than that obtained at $\mathrm{pH}$ 7.0. This indicates that suppression of ionization of

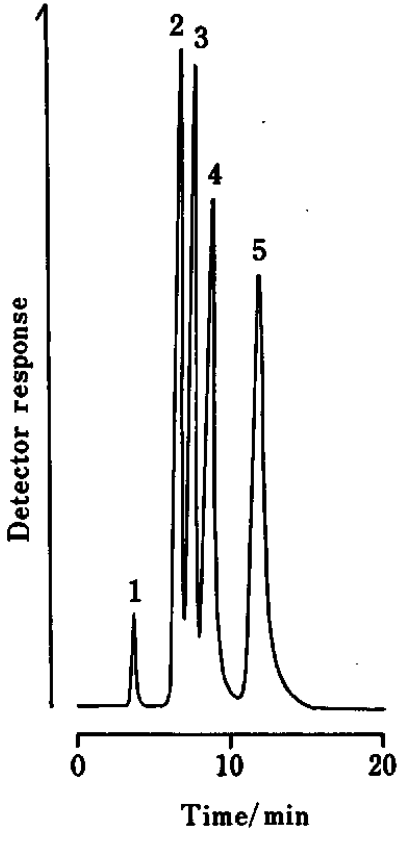

Fig. 2 Chromatogram of five DNS amino acids obtained by the HPLC with fluorescence enhancement detection. The sample solution containing the DNS amino acids was injected into the HPLC system. Peaks: 1, DNS-Lys; 2, DNS-Thr; 3, DNS-Glu; 4, DNS-Ala; 5, DNS-Phe. Amount: 4 pmol each on column. Detector sensitivity: $\times 1$.

amine residue is needed for DNS-Lys to bind with BSA. Further studies on the fluorescence enhancement are now in progress.

\section{Postcolumn HPLC with BSA as a fluorescence enhancement reagent}

From the results described above, BSA was proved to be a fairly selective fluorescence enhancement reagent for some probes such as DNS amino acids. Hence, BSA was used as a fluorescence enhancement and postcolumn reagent for the HPLC determination of DNS amino acids. DNS-L-alanine (DNS-Ala), DNS-Lglutamic acid (DNS-Glu), DNS-Lys, DNS-L-threonine (DNS-Thr) and DNS-L-phenylalanine (DNS-Phe) were selected as model compounds for the HPLC study. These compounds are DNS derivatives of neutral, acidic, basic, hydroxy and aromatic amino acids, respectively. High concentrations of organic solvents such as methanol and acetonitrile could not be used for the mobile phase, since the precipitation of BSA occurred in the HPLC line. Therefore, ion-exchange chromatography was adopted in this study. These five model compounds could be separated within $15 \mathrm{~min}$ on a strong anion exchange column, TSKgel QAE $2 S W(250 \times 4.6 \mathrm{~mm}$ i.d.), by isocratic elution with $50 \mathrm{mM}$ phosphate buffer (pH 2.3); a typical chromatogram is shown in Fig. 2. The effects of the concentration $(0.01-0.5 \mathrm{M})$ and $\mathrm{pH}$ $(2.0-4.0)$ of the phosphate buffer as mobile phase on the separation of DNS amino acids were examined. All 
Table 3 Effect of the length of reaction coil on the peak height

\begin{tabular}{ccc}
\hline Reaction coil/m & Relative peak height & $\begin{array}{c}\text { Retention time/ } \\
\text { min }\end{array}$ \\
\hline 0.9 & 92 & 14.2 \\
3.5 & 93 & 14.7 \\
5.0 & 100 & 14.9 \\
7.0 & 61 & 15.5 \\
\hline
\end{tabular}

The peak height obtained with $5 \mathrm{~m}$ was taken as 100 .

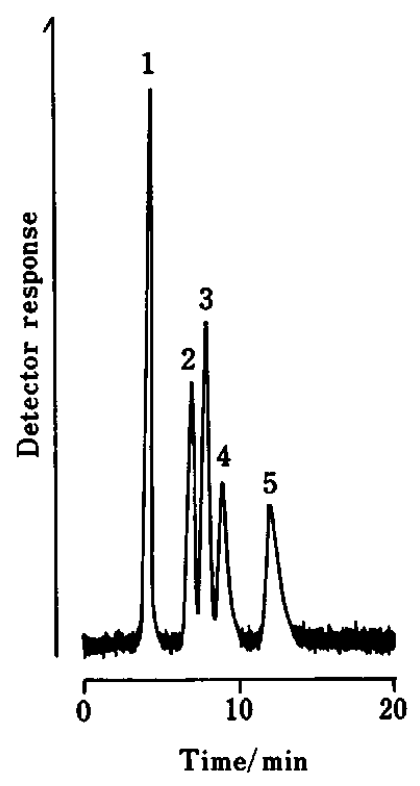

Fig. 3 Chromatogram of five DNS amino acids obtained by the HPLC with conventional fluorescence detection. The same sample solution as in Fig. 2 was subjected to the HPLC system. The HPLC system was the same as described under Experimental except that the BSA solution was replaced with $100 \mathrm{mM}$ phosphate buffer (pH 9.0). Peaks: as in Fig. 2. Detector sensitivity: $\times 32$.

DNS derivatives decreased their retention times with increasing the concentration and decreasing the $\mathrm{pH}$ value; $50 \mathrm{mM}$ phosphate buffer ( $\mathrm{pH} 2.3$ ) was employed as optimum for the mobile phase. Base line and rapid separation of these derivatives could not be obtained with weak anion-exchange columns (TSkgel DEAE-NPR and TSKgel DEAE-5PW).

The effects of the $\mathrm{pH}$ and of the concentration of phosphate buffer, which was used for preparing the BSA solution as a postcolumn reagent, on fluorescence development were examined. All of the compounds tested afforded almost maximum and constant peak heights when phosphate buffer solutions in the $\mathrm{pH}$ range of $8.8-10.0$ were used. Concentrations of phosphate in the range $70-150 \mathrm{mM}$ in the BSA solution provided maximum and constant peaks from tested compounds. Therefore, $100 \mathrm{mM}$ phosphate buffer (pH 9.0) was selected for the recommended procedure. The $\mathrm{pH}$ of

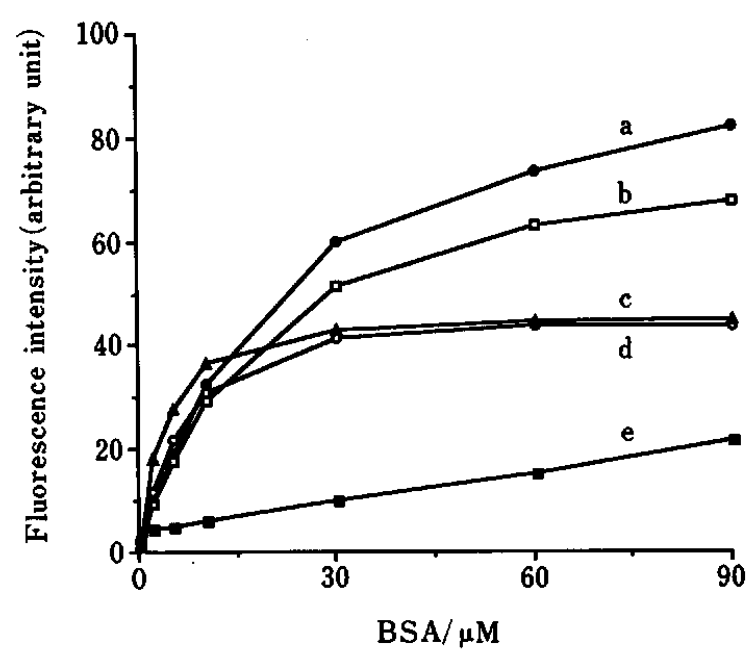

Fig. 4 Effect of BSA concentration on the peak heights of DNS amino acids. Curves: a, DNS-Thr; b, DNS-Glu; c, DNS-Phe; d, DNS-Ala; e, DNS-Lys.

the final reaction mixture was almost 6.5 .

The concentration of BSA had an effect on fluorescence development (Fig. 4). The peak heights of the compounds were getting higher as the BSA concentration was increased. However, the precise measurement was inhibited by the evolution of gas in the reaction coil at BSA concentrations above $70 \mu \mathrm{M} ; 60 \mu \mathrm{M}$ BSA was selected for the recommended procedure.

Table 3 shows the effect of reaction coil length on the fluorescence intensity of DNS-Ala. The peak height was almost maximum at the tube length between 0.9 and $5.0 \mathrm{~m}$. On the other hand, the height decreased at $7.0 \mathrm{~m}$ because of the diffusion; a $5 \mathrm{~m}$ reaction coil was adopted to yield the highest peaks.

In order to evaluate the sensitivity of the method, the same sample as in Fig. 2 was analyzed by the HPLC with conventional fluorescence detection, measured at an excitation wavelength of $330 \mathrm{~nm}$ and an emission wavelength of $560 \mathrm{~nm}$, based on the fluorescence of the intact DNS derivatives (Fig. 3). The analysis was carried out by using $100 \mathrm{mM}$ phosphate buffer (pH 9.0) instead of the BSA solution as the postcolumn reagent (fluorescence enhancer) solution. Detection limits for DNS derivatives obtained by the fluorescence enhancement detection were 169 (DNS-Lys), 34 (DNS-Thr), 42 (DNS-Glu), 59 (DNS-Ala) and 59 (DNS-Phe) fmol/ injection, at a signal to noise ratio of 3 , respectively. These values were 8-109 times lower than those obtained with the conventional fluorescence detection. Calibration graphs for DNS derivatives were linear up to at least $50 \mathrm{pmol}$ on column each. The correlation coefficients were above 0.998 for all the compounds tested. The precision was established by repeated determination $(n=10)$ of the DNS derivatives (4 pmol each); the relative standard deviations for all the compounds did not exceed $4.8 \%$. 
In conclusion, BSA was evaluated as a fluorescence enhancement reagent. The degree of the fluorescence enhancement by BSA depended on the structures of basic skeletons and side-chains. BSA was found to be a fairly selective fluorescence enhancement reagent for some compounds such as DNS amino acids. Moreover, BSA can be applied for the determination of DNS amino acids by the postcolumn HPLC with fluorescence enhancement detection. DNS derivatives of amino acids can be measured 8-109 times more sensitively with the enhancement detection method than the conventional fluorescence detection. Although the method using BSA has limitations in the use of organic solvents for the mobile phase, it should be useful in cases of ion-exchange and gel-permeation chromatographic modes.

\section{References}

1. Y. Ohkura, M. Kai and H. Nohta, J. Chromatogr., 659, 85 (1994).

2. R. W. Frei and J. F. Lawrence, "Chemical derivatization in analytical chemistry (I-2)”, Plenum Press, New York, 1981.

3. H. J. E. M. Reeuwijk, H. Irth, U. R. Tjaden, F. W. H. M. Merkus and J. Greef, J. Chromatogr., 614, 95 (1993).

4. R. P. Frankewich, K. N. Thimmaiah and W. L. Hinze, Anal. Chem., 63, 2924 (1991).

5. M. Yamaguchi, K. Wada, J. Ishida and M. Nakamura, Analyst [London], 117, 1859 (1992).

6. L. Stryer, J. Mol. Biol., 13, 482 (1965).

(Received May 1, 1995)

(Accepted June 28, 1995) 